\begin{abstract}
MS24 P01
Ionic path in oxygen-ionic conductor $\mathbf{L a}_{9.70}\left(\mathbf{S i}_{5.8} \mathbf{M g}_{0.2}\right) \mathbf{O}_{26.35} \quad$ Yoshitaka Matsushita ${ }^{\mathrm{a}}, \quad$ Ali Roushown ${ }^{\text {a }}$, Fujio Izumi ${ }^{a}$, Hideaki Kitazawa ${ }^{a}$, Masatomo Yashima ${ }^{\mathrm{b}}$ a Quantum Beam Centre, NIMS, Tsukuba, Japan ${ }^{\mathrm{b}}$ Tokyo Institute of Tech, Yokohama, Japan E-mail: MATSUSHITA.Yoshitaka@nims.go.jp
\end{abstract}

Keywords: Ionic conductor, Electron distribution, Maximum-entropy method

Oxygen-ionic conductors are very important materials for various kinds of applications such as fuel cells and oxygen-gas sensors. In 1995, Nakayama found new type of oxygen-ionic conductor; Ln-Si-O apatites ( $\mathrm{Ln}=$ Lanthanoids). [1,2] These compounds show better oxygen ionic conductivity in middle range of temperature compared with other famous oxygen-ionic conductors $\left(\mathrm{ZrO}_{2}\right.$ and/or $\mathrm{CeO}_{2}$-based materials). Recently, Yoshioka reported that Mg-doped apatites show the best ionic conduction. [3] However, in all of the apatite system, details of mechanism of ionic conduction are still unknown.

Therefore, we carried out high-temperature diffraction study using synchrotron radiation to suppose mechanism from a change of electronic density. In this study, we selected $20 \% \mathrm{Mg}$-doped compound, which showed the highest conductivity. Intensity data was measured by powder method at BL-19XU beam line, SPring-8, Japan, and the temperature range was from room temperature to $1283 \mathrm{~K}$. The crystal structures were refined by Rietveld method using RIETAN-FP [4], the electron distributions were investigated by whole-pattern fitting approach based on the maximum-entropy method (MEM) using PRIMA [5], and the structure and electron distributions were drawn by VESTA $[6,7]$.

In whole temperature range, this compound belongs to $\mathrm{P}_{3} / \mathrm{m}$. The Rietveld refinement suggested an interstitial oxygen site $\mathrm{O} 5$ located at $(-0.01,-0.003,-0.002)$. Electron density map derived from the MEM analyses revealed that oxide ions $\mathrm{O} 4$ located at 2a site were diffused along the $c$ axis. In addition, a short-range curved diffusion path through the interstitial site was clearly visualized between two oxygen atoms $\mathrm{O} 3$ within the $\mathrm{SiO}_{4}$ tetrahedra.

[1] Nakayama S., Kagayama T., Aono H., Sadaoka, Y., J. Mat. Chem., 1995, 5, 1801.

[2] Nakayama S., Aono H., Sadaoka, Y., Chem. Lett., 1995, 24, 431.

[3] Yoshioka, H., Chem. Lett., 2004, 33, 392.

[4] Izumi F., Dilanian R. A. in "Recent Research Developments in Physics" Transworld Research Network Vol. 3", Trivandrum, 2002, 699.

[5] Momma K., Izumi, F., IUCr Newslett., 2006, 106.

[6] Izumi, F., Momma K., Solid State Phenom., 2007, 130, 15.

\section{MS24 P02}

Experimental charge density study of (E)-4-(2,4diisopropylphenyl)-4-oxo-2-butenoic acid

Sladjana B. Novaković ${ }^{a}$, Goran A. Bogdanović ${ }^{a}$, Branko J. Drakulić $^{b}$, Anne Spasojević-de Biréc, Ivan O. Juranić ${ }^{d}$ ${ }^{a}$ VINČA Institute of Nuclear Sciences, Laboratory of Theoretical Physics and Condensed Matter Physics, P.O. Box 522,11001 Belgrade, Serbia. ${ }^{b}$ Department of Chemistry, Institute of Chemistry, Technology and Metallurgy, University of Belgrade, Njegoševa 12, 11000 Belgrade, Serbia. ${ }^{2}$ Laboratoire SPMS, UMR 8580 CNRS, Ecole Centrale Paris, 92295 Châtenay-Malabry, France.
${ }^{d}$ Faculty of Chemistry, University of Belgrade, P.O. Box 158, 11001 Belgrade, Serbia.

E-mail: snovak@vin.bg.ac.yu

Keywords: 4-aryl-4-oxo-2-butenoic acids, electron density, electrostatic properties

4-Aryl-4-oxo-2-butenoic acids (AAA) exert antibacterial and antineoplastic activity [1]. The (E)-4-(2,4diisopropylphenyl)-4-oxo-2-butenoic acid was a precursor of one of most active CSAB derivative [2] that selectively suppress proliferation of neoplastic $\mathrm{HeLa}$ cells in submicromolar concentration. The activity of AAA are ascribed to high affinity of their ketovinyl moieties ($\mathrm{C}(\mathrm{O})-\mathrm{CH}=\mathrm{CH}-)$ toward octagonal thiol groups of biomolecules. Introduction of branched alkyl chains on phenyl ring significantly increases activities within the congcneric group of compounds. Here, we present the results of the experimental charge density study of the $(E)$ 4-(2,4-diisopropylphenyl)-4-oxo-2-butenoic acid. The Hansen-Copens multipole model was used to describe the electron density distribution within this compound. Subsequently, the topological analysis was performed to characterize the properties of the covalent bonds and interactions. The values of $\rho$ in the sixteen $\mathrm{C}-\mathrm{C}$ bonds are ranging from 1.64-2.46 e. $\AA^{-3}$. The highest value is found for the formally double bond of the ketovinyl moiety. The strongest interaction in the structure is the cyclic O-H...O hydrogen bond formed between the carboxyl moieties (Scheme). In the aim to understand the further reactivity of this molecule the molecular electrostatic potential was also determined.

[1] a) Z. Juranić, Lj. Stevović, B. Drakulić, T. Stanojković, S. Radulović, I. Juranić, J. Serb. Chem. Soc. 64 (1999) 505; b) K. Bowden, A. DelPozzo, C.K. Duah, J. Chem. Res. (1990) (S) 377, (M) 2801 .

[2] B.J. Drakulić, Z.D. Juranić, T.P. Stanojković, I.O. Juranić, J. Med.Chem. 48 (2005) 5600.

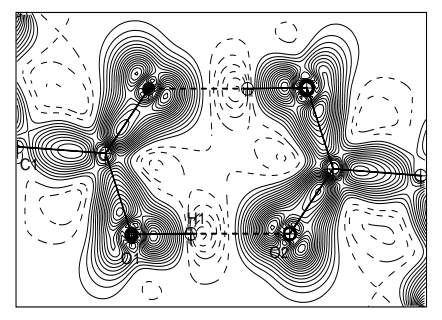

\section{MS24 P03}

Crystal geometry and scale levels of plastic flow localization in metals and alloys Lev B. Zuev, Svetlana A. Barannikova Institute of Strength Physics and Materials Science, SB RAS, Tomsk, Russia.

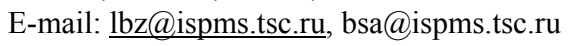

Keywords: single-crystal orientation, crystal geometry, wavelength

Using a holographic technique, plastic flow investigations were carried on for FCC, BCC and HCP monocrystals tested in tension. The experimental evidence obtained points to the existence of hitherto unknown wave processes involved in deformation localization. Waves of this kind are generated in the deforming crystals as an ensemble of localized plastic deformation nuclei moving in a concerted manner and evolving with time. Wave 
pattern type observed at a given flow stage is determined by the acting law of work hardening $\theta(\varepsilon)$ (here $\theta \equiv \mathrm{G}^{-}$ ${ }^{4} \cdot \mathrm{d} \sigma / \mathrm{d} \varepsilon$ is the coefficient of deformation hardening; $\sigma$ is the plastic flow stress; $\varepsilon$ is the deformation and $G$ is the shear modulus. By addressing plasticity phenomena, a prerequisite for wave model development is the establishment of a relationship between the observed localization patterns on the one hand and the material lattice parameters and the crystallographic aspects of plastic flow, i.e. extension axis orientation in single crystals, Schmidt factor value, acting slip and twinning systems, on the other. A detailed study of plastic deformation was made on a range of polycrystalline materials and single crystals of pure copper and nickel, Fe$3 \% \mathrm{Si}$ alloy, chromium and nickel based austenite $\left(\gamma-\mathrm{Fe}_{\mathrm{I}}\right.$ containing $0.35 \% \mathrm{Ni}$ and $0.5 \% \mathrm{Ni}$ in $\gamma$-solid solution) and high manganese austenite $\left(\gamma-\mathrm{Fe}_{\mathrm{II}}\right.$ containing $0.93 \% \mathrm{C}$ and $1.03 \% \mathrm{C})$. Variation in the nitrogen and carbon contents and in extension axis orientation causes a changeover in the type of shear processes (single/multiple slip) and in the mechanism involved in the deformation (dislocation glide/twinning).

The following quantitative characteristics have been defined for the waves in question: wavelength $5 \leq \lambda \leq 10$ $\mathrm{mm}$; velocity $10^{-5} \leq \mathrm{V} \leq 10^{-4} \mathrm{~m} / \mathrm{s}$ and frequency $10^{-3} \leq \omega \leq$ $10^{-2} \mathrm{~Hz}$. An analysis of the spatial characteristics of localized plastic flow waves suggests that (i) macroscopic localization zones emergent in a deforming monocrystal are oriented in space with respect to its extension axis depending on its crystallographic parameters; on the observation plane these coincide with the lines of acting slip/twinning systems having maximal Schmidt factor values;

(ii) the number of localized plastic deformation nuclei acting at the easy glide stage in a deforming monocrystal is defined by the number of glide/twinning systems acting at a given crystallographic orientation;

(iii) correlation exists between the quantities $2 \pi / \mathrm{k} \cdot \mathrm{V}_{\mathrm{aw}}=$ $\lambda \cdot V_{\text {aw }}$ (here $k=2 \pi / \lambda$ is the wave number) and $d \cdot V_{\perp}$ (here $d$ is the spacing between closely packed planes in a single crystal and $\mathrm{V}_{\perp}$ is the velocity of transverse ultrasound waves).

Matching of the latter quantities obtained for metals investigated reveals that they are very close, with the average ratio $\left\langle 2 \lambda \cdot \mathrm{V}_{\mathrm{aw}} / \mathrm{d} \cdot \mathrm{V}_{\perp}\right\rangle$ being $0.96 \approx 1$, i.e. $\lambda \approx$ $\mathrm{d} \cdot\left(\mathrm{V}_{\perp} / \mathrm{V}_{\mathrm{aw}}\right)$. In view of the fact that $\mathrm{d} \approx \mathrm{b}$ (Burgers vector), the above can be considered as evidence for the existence of relationship between the micro- and macroscopic scale parameters of plastic deformation. Thus, the micro- and macro-scale parameters of plastic flow in single-crystal and polycrystalline materials are related by a simple relationship $\lambda \cdot \mathrm{V}_{\mathrm{aw}} \approx 1 / 2 \mathrm{~d} \cdot \mathrm{V}_{\perp}$. The slip lines are distributed inhomogeneously over the monocrystal since they are concentrated in the nuclei of deformation localization. 